\title{
La convention 210 du conseil de l'Europe (2011) sur la prévention et la lutte contre la violence à l'égard des femmes et les violences domestiques
}

\author{
Convenção 210 do conselho da europa (2011) sobre a prevenção e a luta contra a \\ violência contra as mulheres e a violência doméstica \\ Convention 210 of the council of europe (2011) on the prevention and combat violence \\ against women and domestic violence
}

\author{
Virginie Saint-James \\ FDSE/OMIJ \\ Université de Limoges, France
}

\begin{abstract}
Résumé
Le 7 avril 2011, le Conseil de l'Europe adopte une Convention sur la prévention et la lutte contre la violence à l'égard des femmes et la violence domestique. Il s'agit d'un instrument régional de protection des droits de l'homme. Le texte de la convention déploie un discours assez traditionnel sur les violences domestiques qu'il ne définit guère si ce n'est par l'expression : « au sein de la famille ou du foyer ou entre anciens et actuels conjoints ou partenaires, indépendamment du fait que l'auteur de l'infraction partage ou a partagé le même domicile que la victime ». Mais la Convention se distingue surtout par le fait qu'elle rompt avec les textes européens actuels en obligeant les Etats à utiliser avec une certaine sévérité le droit pénal pour réprimer ces violences, au besoin en développant toutes les formes possibles de compétence pénale des Etats, jusqu'à des possibilités d'extradition.

Mots-clés: Violences; Femmes; Europe; Droits de l’homme; Droit pénal.
\end{abstract}

\begin{abstract}
Resumo
Em 7 de abril de 2011, o Conselho da Europa aprovou uma convenção sobre a prevenção e o combate à violência contra as mulheres e a violência doméstica. Ele trata de um instrumento regional para a proteção dos direitos humanos. O texto da convenção implanta um discurso bastante tradicional sobre a violência doméstica que dificilmente definida, exceto pela frase "dentro da família ou unidade doméstica ou entre antigos e atuais cônjuges ou companheiros, independentemente do autor da infração compartilhar ou de ter compartilhado o mesmo domicílio que a vítima". Mas a Convenção é particularmente notável na medida em que rompe com as normas europeias em vigor, obrigando os estados a utilizar, com certo rigor o direito penal para conter essas violências, sendo necessário para o desenvolvimento de todas as formas possíveis de jurisdição penal dos Estados, até a possibilidade de extradição.

Palavras-chave: Violência; Mulheres; Europa; Direitos humanos; Direito penal.
\end{abstract}

\begin{abstract}
On April 7, 2011, the Council of Europe adopted a Convention on preventing and combating violence against women and domestic violence. It is a regional instrument for the protection of human rights. The text of the convention deploys a fairly traditional discourse on domestic violence that hardly defined, except for the phrase "within the family or domestic unit or between former and current spouses or partners, regardless of the offender share or have shared the same household as the victim." But the Convention is particularly notable in that it breaks with the European standards, forcing the states to be used with some accuracy the criminal law to address such violence, being necessary to the development of all possible forms of criminal jurisdiction of the United until the possibility of extradition.

Key-words: Violence; Women; Europe; Human rights; Criminal law.
\end{abstract}

Le 7 avril 2011, le Conseil de l'Europe adopte une Convention sur la prévention et la lutte contre la violence à l'égard des femmes et la violence domestique. Il s'agit d'un instrument régional ouvert à la ratification des membres du Conseil, mais aussi des Etats non membres, singulièrement ceux qui ont participé à son élaboration (Canada, USA, Japon, Mexique, Saint Siège).

Ces questions des violences faites aux femmes ne sont pas nouvelles pour l'Europe des droits de l'Homme. Dans le prolongement d'une Recommandation de 2002 (Rec. (2002) 5 du 30 avril 2002) qui a servi de guide et d'inspiration, le Conseil entre 2006 et 2008 a en effet mené une campagne pour lutter contre ces formes de violence. Le comité des ministres a institué en 2008 un comité ad hoc (CAHVIO) qui, à son tour, a élaboré en 2009 un projet de convention. Il est établi avec l'aide de représentants des Etats, d'ONG et d'organisations internationales. Le projet est alors finalisé en 2010 et soumis à l'Assemblée parlementaire pour avis (Avis 280 (2011). Le 11 mars 2011, cet avis positif permet l'adoption d'une convention portant actuellement la signature de 17 Etats.

Ce nouveau traité international prend sa place dans un contexte normatif international assez dense, notamment depuis que l'ONU s'est saisie de ces questions dans la Convention des Nations unies 
de 1992 sur l'élimination de toutes les formes de discrimination à l'égard des femmes (CEDEF). Il tient compte, par ailleurs, des avancées du droit international pénal incriminant le viol en temps de guerre, par une référence au statut de la Cour pénale internationale. Plus large que plusieurs autres textes internationaux portant sur un sujet similaire (Convention interaméricaine pour la prévention, la sanction et l'élimination de la violence contre la femme (1994, Organisation des états américains), Protocole à la Charte africaine des droits de l'homme et des peuples relatifs aux droits des femmes (Union africaine 2003,), la convention 210 tend à renforcer l'action des Etats signataires.

Adoptée discrètement et peu commentée par les juristes (W. H., RGDIP, 2012) elle est censée devenir un texte contraignant pour les Etats qui la ratifieront. L'impression initiale qu'elle produit est double. Si l'on peut louer la volonté d'établir une répression des violences par le droit, on ne peut manquer de s'interroger sur une certaine tardiveté, face à un type de violence à la fois courant et connu de tous. C'est qu'il faut rappeler quelques préalables quant à l'intervention du droit dans le domaine des violences domestiques.

Est-il en effet légitime pour la puissance publique de se saisir des violences de l'intimité ? La question est posée de la vie privée face à l'action de l'Etat. Historiquement, la vie privée et familiale a fait l'objet d'une protection qui est parfois, notamment dans les pays anglo-saxons, au départ même de l'affirmation des droits. Son opposabilité à la puissance publique est une valeur cardinale que le droit international des droits de l'Homme consacre et développe par exemple par l'article 12 de la Déclaration universelle des droits de l'homme ou l'article 8 de la Convention européenne des droits de l'homme. Il est donc difficile pour l'Etat démocratique, garant de l'application de tels droits, de justifier une immixtion dans cette sphère.

Une seconde difficulté fondamentale réside dans l'émergence d'un secteur des droits de l'Homme spécifique au genre. En effet le paradigme initial, plus encore en droit international qu'ailleurs, est d'affirmer leur universalisme. La catégorisation est souvent présentée comme une erreur conceptuelle (Sudre, 2008, p. 43). Or, les violences familiales fréquemment assimilées aux "femmes battues », posent un problème au juriste des droits humains.

Ajoutons encore que nous rencontrons ici un scepticisme démobilisateur. Les violences familiales ont longtemps fait l'objet d'un discours de déni, les ramenant soit à des relations interindividuelles complexes, soit aux conséquences de problèmes sociaux collectifs Insérant ces violences dans le cadre de la lutte contre la pauvreté ou contre l'alcoolisme ; si bien que le droit doute encore de la pertinence de son intervention dans le cadre interne - a fortiori dans le cadre international où on peut le trouver très éloigné de son objet.

D'autres obstacles peuvent aussi déterminer l'abstention de la puissance publique. Insérées dans la construction sociale, les pratiques familiales bénéficient de la protection qui s'attache aux systèmes culturels. Ainsi le « crime d'honneur», le « viol entre époux», ou les « mutilations sexuelles » peuvent en droit international être rattachés à la souveraineté de l'Etat qui comporte le droit de choisir son système politique et social et se trouver ainsi protégés par le principe de non-ingérence.

Enfin l'inertie des Etats en droit interne comme en droit international peut aussi provenir de l'ignorance, faute de quantification (Conseil de l'Europe FLYER 2010), ou du découragement, faute de disposer des réponses adéquates. Selon le Conseil de l'Europe « Entre un cinquième et un quart des femmes subissent des violences physiques une fois au moins au cours de leur vie adulte et plus d'une femme sur dix a déjà souffert d'abus sexuel avec usage de la force. Entre 12 et $15 \%$ des femmes vivent une relation marquée par des violences domestiques après l'âge de 16 ans. Bien d'autres continuent de subir les violences physiques et sexuelles d'anciens partenaires ». Longtemps, la seule réponse des victimes à la violence ne pouvait être que la fuite du domicile, totalement inadaptée à leurs problèmes de sécurité et aux questions de dépendance économique.

La convention de 2011, en franchissant ces obstacles, propose donc aux Etats qui la ratifieront de s'engager de façon contraignante, au-delà des recueils apitoyés de vœux pieux que sont habituellement les textes de la soft law internationale dans ce domaine. Le texte envisage les violences sur le genre de façon très générale, mais il nous retiendra ici pour son apport en matière de violence domestique. Résultat d'un large consensus dans la rédaction, le texte développe à ce propos un discours juridique ambigu car fondé sur des difficultés de définitions initiales (I). Mais il développe des obligations étatiques très larges dont l'avenir dira si elles sont irréalistes (II).

\section{I- Le discours conventionnel sur les violences de l'intimité}

La Convention ne peut éviter de préciser son champ d'application et, ce faisant, ne peut manquer de définir les victimes et les violences. Mais si la méthodologie est inévitable, le résultat est ambigu, car encore doublement empreint des contraintes conceptuelles et politiques des rédacteurs du texte, face aux aspects sexués de la question (A) et de la difficulté à identifier la singularité de ce type de violences $(\mathrm{B})$.

A- La difficulté à identifier les victimes des violences intimes

Le droit international des droits de l'Homme est essentiellement né après la Seconde guerre 
mondiale et il s'est immédiatement trouvé en butte aux conséquences de la Guerre froide et de la décolonisation. Il n'a pu faire sa place dans ces tendances de fond qu'en affirmant à la fois l'universalisme des droits et leur objectivité. Sur ces principes, il est difficile de rédiger des textes spécifiques aux femmes (1). Dès lors que ce premier obstacle est surmonté ou contourné, reste à trancher la question des violences visées et de leur singularité intrinsèque, ce que la convention peine à faire (2).

1- Une norme sexuée en matière de violence domestique?

Construire une convention qui vise particulièrement les femmes est en soit un danger pour l'universalisme des droits de l'Homme. Face à ce problème, le droit international général a trouvé la parade : convenir que certaines violations des droits touchent majoritairement les femmes. Il faut alors convenir au surplus que cette surreprésentation est le produit de causes structurelles qui gisent dans une discrimination fondée sur une représentation des rôles en fonction des genres, ce qui permet l'appréhension par les droits de l'Homme à travers le prisme de la non-discrimination.

Au plan universel, en 1992, le Comité pour l'élimination de la discrimination envers les femmes au sein de l'ONU a contribué par une Recommandation générale sur la violence à l'égard des femmes à la faire reconnaître comme une discrimination. En 1993, l'Assemblée générale des Nations unies a adopté une Déclaration sur l'élimination de la violence à l'égard des femmes. Ce but fut intégré dans les objectifs de la conférence de Beijing en 1995 et fera sans doute partie des discussions d'une future grande conférence envisagée pour 2015.

Telle fut également la démarche suivie en Europe. Le comité directeur pour l'égalité entre les femmes et les hommes (CDEG) a contribué à faire naître en 2002 une Recommandation (précitée) «sur la protection des femmes contre la violence » porteuse d'une stratégie globale de tous les Etats. Le 10 décembre 2008, les délégués des ministres du Conseil de l'Europe ont donné mandat au Comité ad hoc pour rédiger un texte afin de prévenir et combattre la violence domestique, y compris les formes spécifiques de violence à l'égard des femmes, et pour protéger et soutenir les victimes des tels actes de violence et poursuivre leurs auteurs.

Ce texte s'inscrit dans la lignée des normes contre les violences fondées sur le genre et repose conceptuellement sur la nécessité d'une lutte contre la discrimination. Ainsi son Préambule aux alinéas 10, 11 et 13, établit-il un lien entre la réalisation de l'égalité des sexes et l'éradication de la violence en posant « la nature structurelle » de la violence à l'égard des femmes comme « fondée sur le genre », à laquelle les femmes et les filles « sont plus exposées que ne le sont les hommes ». Il est alors intellectuellement possible de rédiger ce texte spécifique aux femmes sans violer l'universalisme, et même en s'en réclamant.

Mais cette spécificité sexuée du texte est-elle tenable en matière de violence domestique ?

2- Les victimes de violences domestiques dans la convention

En quête de définition de la violence, le texte se réfère à une approche pragmatique. Il s'agit d'une méthode usuelle des travaux du Conseil de l'Europe. Par exemple, dans l'Exposé des motifs (Conseil de l'Europe, 15 mars 2000, Doc. 8667, Rapport de la Commission sur l'égalité des chances pour les femmes et les hommes, la violence représente une " violation générale des droits humains, droit à la vie, à la sécurité, à la dignité et à l'intégrité physique et mentale ", ce qui est loin d'être une définition évidente et plutôt une définition opérationnelle.

Mais la convention est censée ne viser que la violence à l'égard des femmes, en introduisant un rappel fréquent de la disproportion du nombre de victimes féminines. Si l'économie générale du texte se comprend, la question n'en est pas moins délicate dès lors que l'on aborde les violences domestiques. Certes, selon l'alinéa 15 du Préambule de la Convention, « la violence domestique affecte les femmes de manière disproportionnée » et il n'est pas possible de la laisser en dehors du champ de la convention. Mais il n'est pas davantage possible d'affirmer que ce type de violence n'affecte exclusivement que des femmes. Aussi, la convention est-elle applicable aux hommes dont elle reconnaît qu'ils "peuvent être également victimes de violences domestiques" (ibid). Cette extension permet aussi d'étendre la protection du texte par l'article 16 du Préambule aux victimes par ricochet que sont parfois les enfants en tant que témoins Il a sans doute été difficile d'arriver à une telle rédaction à la fois cohérente et englobante.

Une question demeure: dans les projets intermédiaires, les personnes âgées et parfois les personnes handicapées étaient citées au titre des victimes potentielles de la violence domestique. Elles ont disparu du texte sans que le rapport explicatif n'en donne la raison, sauf à souligner que les données en ce domaine sont "relativement rares". Seule l'Assemblée du Conseil de l'Europe semble s'en être émue. Tout en saluant la définition englobante des victimes qui fut retenue, elle remarque cette carence. L'Assemblée est tout particulièrement préoccupée par le caractère insuffisant de la protection de certains groupes vulnérables particuliers, tels que les enfants, les personnes âgées et les femmes migrantes n'ayant pas de statut de résident régulier. Ces femmes ne sont prises en compte que si elles perdent leur statut de résident après la fin de leur relation avec leur conjoint ou compagnon, en raison des violences qu'elles ont subies. L'institution propose des protocoles particuliers pour y remédier. En outre, afin d'aborder la situation et les besoins de groupes vulnérables particuliers, l'Assemblée invite le Comité 
des Ministres à envisager l'ajout de trois protocoles additionnels au projet de convention, concernant respectivement les enfants, les personnes handicapées et les personnes âgées

Cette recommandation illustre l'une des difficultés des droits internationaux catégorisés : le risque de multiplication des textes, donc de perte de sens et de cohérence de l'action internationale en matière de droits de l'Homme qui en résulte. Toujours est-il que, selon l'article 2, « les parties sont encouragées à appliquer la convention à toutes les victimes de violence domestique. " A condition de l'identifier et de savoir ce que ce terme recouvre.

B- L'absence de singularité conventionnelle des violences domestiques

Dès le premier article, on ne peut manquer d'éprouver l'impression de la lecture d'un catalogue de violences et de mesures disparates, au sein desquels la violence domestique ne trouve guère sa spécificité que par un mécanisme de « sur-définition » particulier (1) et qui ne s'explique que par une volonté de partage d'expérience (2).

1- Le mécanisme conventionnel de définition

La convention choisit d'abord l'approche sexuée en décrivant les violences à l'égard des femmes comme "fondées sur le genre ». Elles peuvent être de nature " physique, sexuelle, psychologique ou économique, y compris la menace de se livrer à de tels actes, la contrainte ou la privation arbitraire de liberté, que ce soit dans la vie publique ou privée $»$.

La convention sur-définit ensuite la violence domestique dans l'article $3 \mathrm{~b}$ comme : « tous les actes de violence physique sexuelle psychologique ou économique qui surviennent au sein de la famille ou du foyer ou entre anciens et actuels conjoints ou partenaires, indépendamment du fait que l'auteur de l'infraction partage ou a partagé le même domicile que la victime ».

Cette méthode ne permet guère de déterminer les actes de violence domestique, et elle ne permet pas davantage de cerner les obligations des Etats en ce domaine. En fait, l'économie générale du texte repose sur les comportements définis par les articles 33 et suivants qui en constituent la substance matérielle. Y sont stigmatisés les violences psychologiques, harcèlement, violence physique, violence sexuelle y compris le viol et singulièrement le viol entre époux, mariages forcés, mutilations génitales féminines; avortement et stérilisations forcés, harcèlement sexuel. Le texte vise aussi, dans l'article 42, les mêmes comportements lorsqu'ils sont soit disant justifiés par la culture, la coutume, la religion ou le « prétendu honneur ».

La convention permet alors de déduire que ces actes prennent la qualification de « violence domestique " lorsqu'ils sont commis dans le cadre décrit par l'article $3 \mathrm{~b}$. Si le texte permet effectivement de couvrir l'ensemble des violences visées dans les travaux des ONG, on peut sans doute lui reprocher son manque de lisibilité immédiate. Par exemple, il faut croiser l'article 36 avec une allusion du Préambule à la jurisprudence de la Cour européenne des droits de l'homme, pour postuler l'exclusion de l'exigence de la preuve d'une résistance physique, pour que la victime puisse invoquer le viol entre époux En souhaitant donc que les Etats tiennent compte de la jurisprudence de la Cour EDH ( M.C. contre Bulgarie).Si le système proposé permet donc une relative exhaustivité, il n'est pas aisé d'en déduire une définition des comportements de violence domestique. C'est que tel n'est peut-être pas son propos principal. Sa conception utilitariste immédiate est de permettre un partage d'expérience (notamment juridique) qui constitue son principal apport en comparaison avec des textes de soft law précédents.

2- La généralisation des avancées vers un standard européen

Les textes internationaux et nationaux en matière de violences domestiques ne manquent pas (Assemblée générale des Nations unies, 2 août 2010, RES 65/208,) En revanche, il est sans doute temps d'uniformiser le droit qu'ils génèrent. Pour cela, la convention 210 est l'un des rares outils contraignants et participe à la technique du standard européen, plus fréquemment rencontrée dans le droit jurisprudentiel, et qui vise à aligner les garanties juridiques offertes par les Etats membres du Conseil de l'Europe, notamment en condamnant à l'évolution des législations désuètes ou isolées.

Le texte évoque donc des pistes d'évolution qui résultent des législations et pratiques le plus en pointe : par exemple en son article 42-2, en instaurant des peines d'incitation des mineurs à commettre des «crimes d'honneur Il vise des actes violents commis par des personnes n'ayant pas atteint l'âge de la majorité pénale à l'instigation des adultes. Il s'agit de juguler une pratique familiale qui tend à déléguer des tâches de «punition » aux plus jeunes membres afin de minimiser les sanctions pénales. La convention prend aussi en compte, dans son article 34 , le harcèlement « non sexuel », souvent le fait d'un ancien conjoint ou partenaire et le rapport explicatif inclut dans cette définition les actes commis sur des espaces virtuels à travers la notion de communication non désirée Elle incite aussi dans son article 33 à introduire les violences psychologiques au sein des violences familiales. Enfin, en visant les actes de complicité ou tentative dans l'article 41, elle tend à sanctionner plus efficacement les tiers prêtant leur concours à des mariages forcés ou des mutilations génitales.

Mais la convention n'a sans doute pas la même efficacité contraignante qu'un arrêt de la Cour européenne des droits de l'homme. Comme toute convention internationale multilatérale, la Convention 210 permet aux Etats d'assortir leur engagement de réserves. Même si la clause d'autorisation de 
l'article 782 et 3 est limitée et ne peut jouer sur le droit matériel conventionnel, les Etats conservent la possibilité de réserver les particularités de leur système de procédure pénale, ce qui n'est pas sans revenir gravement sur les droits des victimes. Ainsi les Etats de Common law pourront-ils, grâce à l'article 44 3 , exprimer leur réticence à l'égard de la compétence pénale personnelle passive, ou leur difficulté à gérer les procédures ex parte et ex officio prises en compte dans l'article 55.

C'est sans doute que le droit pénal est l'enjeu principal de la Convention qui pose essentiellement sur les obligations de criminalisation effective.

\section{II- Les obligations conventionnelles relatives aux violences de l'intimité}

Le texte introduit nombre d'obligations non juridiques au sens strict du terme. Il commence par préconiser des actions qui ne relèvent pas nécessairement de la sphère juridique, telle l'obligation de développer une politique "sensible au genre », ou de collecter des données statistiques. De même une prévention des violences est instaurée ainsi que des devoirs de protection et de soutien des victimes. Il arrive aussi que ces obligations relèvent $\mathrm{du}$ droit, mais pas du droit répressif, par exemple dans l'article 28 en matière de secret professionnel partagé ou bien de droit civil (L'article 32 vise ainsi les conséquences civiles des mariages forcés). Mais il est clair que l'essentiel du dispositif repose sur une criminalisation des violences (A), qui doit garantir une « Europe sans violence domestique » (B).

A- Une obligation générale de pénalisation

L'un des buts essentiels de la Convention 210 est de mettre fin à l'impunité des auteurs de violences domestiques. Sans nier la pertinence de ce dessein, il restera à évaluer la possibilité de le réaliser pleinement par le biais d'une convention internationale supplémentaire.

1- Les obligations pénales des Etats

La convention touche tant le droit pénal de fond que de forme.

Ratione materiae, elle oblige dans son article 29 les Etats à introduire dans leur code pénal les infractions correspondant à ses articles 33 et suivants, en englobant l'instigation parfois ainsi que la complicité et la tentative, sans préjudice d'éventuels recours civils.

Dans le domaine du droit européen conventionnel, l'existence d'obligations positives d'incriminer n'est pas une rareté. Ainsi la Cour européenne des droits de l'Homme a établi des cas où l'Etat a pour obligation positive d'assurer l'effectivité d'un droit par la criminalisation substantielle, par exemple en matière d'esclavage domestique (Sudre F., Marguenaud J.-P., Andriantsimbazovina J., Gouttenoire A., Levinet M. Thémis, 2007). Le texte, tout comme la jurisprudence de la Cour imposent de «promulguer une législation pénale permettant de punir effectivement » (Sudre F., Marguenaud J.-P., Andriantsimbazovina J., Gouttenoire A., Levinet M., p. 175), et non seulement d'inscrire dans le Code pénal une sanction inapplicable ou inappliquée.

Cette obligation d'effectivité débouche alors, comme devant la Cour européenne, sur la nécessité d'établir des punitions effectives, proportionnées et dissuasives selon l'article 45 D'abord en exigeant des Etats qu'ils mettent fin à l'impunité en déployant toutes les possibilités d'une compétence pénale territoriale et personnelle dans l'article 44 Ensuite, bien que le droit européen général recommande de multiplier les alternatives à la prison (Poncella et Roth 2006), la convention 210 n'a de cesse de promouvoir des sanctions pénales et, le cas échéant, « des peines privatives de liberté » par le principe de l'interdiction des modes alternatifs de règlement des conflits

Il est vrai que, par voie de réserve, les Etats peuvent s'en tenir à des sanctions non pénales en ce qui concerne la violence psychologique ou le harcèlement, où il est loisible de s'en tenir à des obligations de suivi et de soins. Mais il s'agit pour l'essentiel d'une obligation de pénalisation qui ne saurait être complète sans un large volet procédural.

Ratione procedere, les nombreuses pressions qui s'exercent, souvent sur les victimes de violences familiales, conduisent à exiger que la poursuite pénale ne dépende pas uniquement de la plainte et que celleci ne soit pas minimisée au profit d'une qualification mineure.

Le texte reprend ensuite de façon très prolixe les conséquences qui découlent de l'obligation "d'enquête effective » en accordant, en amont, une large place aux dispositifs d'aides aux victimes, puis en aval, par des dispositions qui concernent les délais de réponse, les preuves, les mesures de protection et l'aide juridique dans tout le chapitre VI. Toutefois, les particularités procédurales sont telles dans chaque pays d'Europe qu'il est paradoxalement plus difficile de trouver ici un terrain d'entente sur ces questions. Ainsi figurent au paragraphe 2 de l'article 78 des possibilités de réserves sur les points où un accord unanime n'a pu être trouvé. Tel est le cas par exemple dans l'article 58 des règles de prescription.

Surtout, si l'article 30 porte une obligation générale d'indemniser, son paragraphe 2 introduit une intéressante possibilité d'indemnisation par l'Etat, en cas « d'atteintes graves à l'intégrité corporelle ou à la santé, dans la mesure où le préjudice n'est pas couvert par d'autres sources ». On ne s'étonnera guère de voir cet article entrer dans le champ des réserves possibles. Le texte n'en demeure pas moins extrêmement complet et la pertinence de son contenu est évidente. La question demeure toutefois quant à son existence dans l'environnement juridique déjà encombré du Conseil de l'Europe. 
2- La pertinence d'un texte supplémentaire ?

On invoquera volontiers à l'encontre de la Convention 210, les reproches classiques adressés au droit international des droits de l'Homme. Les risques d'atteinte aux caractères d'universalité et d'objectivité ont déjà été mentionnés et ne seront pas repris ici, mais le risque d'atteindre la cohérence de la protection n'est pas négligeable.

La simple lecture du Préambule de la Convention 210 qui met en exergue plus de quinze textes et une référence appuyée à la jurisprudence de Strasbourg en est l'illustration. Attend-on véritablement de la Convention qu'elle s'articule avec l'ensemble de ces références ? On objectera que nombre d'entre elles sont en fait redondantes et inefficaces car dépourvues d'effet obligatoire. Pourquoi alors les mentionner, si la Convention est censée les remplacer? Sans compter qu'il existe, en outre, en fin de convention une clause de compatibilité à l'article 71 qui subordonne ce texte à tous les autres engagements des Etats.

La convention ne peut échapper à la question de son utilité. S'il s'agit de poser l'obligation d'un droit au recours européen efficace en matière de violence domestique, elle existe déjà. La Cour européenne des droits de l'Homme a donné gain de cause à une épouse dont le mari avait tué leurs deux enfants. La requérante a pu établir que lorsqu'elle était venue porter plainte, la police locale l'avait aidée à la modifier en une qualification mineure. En outre, il n'existait pas pour elle de recours en indemnisation. La Cour a évidemment relevé la violation tant de l'article 2 que de l'article 13 de la Convention européenne (Cour EDH, 31 mai 2007, Kontrova c. Slovaquie).

De même, il a déjà été souligné qu'en matière de viol entre époux ou de preuve de résistance au viol, la Cour européenne n'a pas attendu un texte spécifique pour condamner les Etats et les forcer à faire évoluer leur législation.

La Cour a de même, déjà obligé les Etats à faire sortir les violences domestiques de la sphère privée, condamnant la Bulgarie pour ne pas avoir contrôlé un ex-conjoint violent et pour avoir refusé d'ouvrir des poursuites pénales au nom du " caractère privé » de l'affaire (Cour EDH, 12 juin 2008, Bevacqua et S. c. Bulgarie). On pourrait multiplier ainsi les exemples où la Cour développe une jurisprudence qui rejoint les exigences de la convention en termes de harcèlement (Cour EDH, 14 octobre 2010, A. c. Croatie), ou de retrait de plainte (Cour EDH, 9.juin.2009, Opuz c. Turquie), ou de maintien au domicile de la victime (Cour EDH, 15.septembre.2009, E.S. et autres c. Slovaquie).

Demeure aussi posée la question de l'efficience du texte, dépourvu d'effet direct. Son mécanisme de sanction est on ne peut plus classique et repose sur l'institution d'un comité de suivi intitulé " groupe d'experts sur la lutte contre la violence à l'égard des femmes et la violence domestique » Il fonctionnera sur la base des rapports fournis par les Etats quant à leur progrès dans la lutte contre la violence et ses pouvoirs sont peu développés Malgré l'affirmation contraire de l'Assemblée parlementaire. Il s'agit d'un contrôle administratif formel classique qui n'est pas nécessairement dépourvu d'utilité... du moins tant que les Etats sont de bonne volonté....

Sur de telles bases on peut se demander si le texte assurera vraiment une « Europe sans violence domestique $»$.

B- Une Europe sans violence domestique?

Il est permis de douter que le mécanisme conventionnel soit le plus adapté sur le plan matériel et procédural, car il entre en concurrence avec le mécanisme juridictionnel, sans nul doute plus efficace. Mais l'essentiel du texte n'est-il pas d'assurer une réelle coopération pénale internationale et de protéger les victimes d'où qu'elles viennent?

1- Un texte novateur quant à l'exercice international de la compétence pénale.

Si le but du cœur matériel de la convention est le partage d'expérience il n'est pas inutile, mais il n'était pas nécessaire d'élaborer un instrument juridique contraignant. C'est donc que l'apport juridique le plus intéressant réside dans la gestion partagée d'une compétence pénale maximisée.

Alors, l'instrument conventionnel est irremplaçable pour lier les Etats dans la lutte contre l'impunité pénale des violences domestiques. Le texte impose dans son article 21 à un Etat partie de poursuivre les violences commises sur son territoire quelle que soit la nationalité de l'auteur, mais aussi leur ressortissant ayant commis une des infractions à l'étranger ou de protéger des étrangers à l'étranger, s'ils ont leur résidence habituelle sur son territoire.

Le texte fait sauter, de plus, certains verrous du droit de l'extradition, notamment l'exigence de double incrimination, et établit le principe «aut dedere aut judicare » dans les cas de refus d'extrader. Il s'écarte, en outre, des conditions restrictives de plainte de la victime ou d'engagement des poursuites par le parquet de l'Etat où l'infraction a été commise, dans les cas de compétence personnelle active, du fait de la nationalité ou de la résidence habituelle de l'auteur de l'acte.

Devant un tel développement des possibilités de poursuites pénales, l'article $44 \S 6$ doit même prévoir une obligation de consultation entre Etats pour déterminer lequel est le plus en situation d'exercer des poursuites en cas de compétences concurrentes. La convention crée, en outre, dans son article 47, l'un des très rares cas de « récidive internationale » qui n'est guère connue que dans le cadre du faux monnayage en droit communautaire et par la Convention de New York sur les stupéfiants, si bien qu'une infraction à l'étranger devient une circonstance aggravante lors de la réitération dans un autre Etat.

En réalité, la convention est sans doute plus 
un texte sur la fin de l'impunité en cas de violence domestique (et de violence faite aux femmes) qu'une convention de plus, fondée sur le genre. Le texte porte des ambitions très vastes en termes d'application de la loi pénale dans l'espace européen, qu'il tend à sanctuariser à l'abri des violences domestiques.

2- Une Europe refuge?

Le rapport explicatif part du constat que les femmes migrantes sans papiers et demandeuses d'asile forment deux sous catégories " particulièrement vulnérables à la violence sexiste ». Le chapitre VII de la convention demande à leur égard une " compréhension 》 particulière. Dès lors, les Etats doivent développer des politiques d'accueil « sensibles au genre », notamment par des aménagements des centres d'accueil.

Pour les femmes migrantes, 1'article 59 vise à leur faire octroyer un permis de séjour qui leur soit propre et ne dépende pas de celui de leur compagnon en cas de violences. A l'heure actuelle, les Etats imposent généralement une durée de mariage ou de relation de trois ans avant de pouvoir y prétendre.

La convention instaure la nécessité d'un permis autonome d'une durée limitée. Une telle attitude peut évidemment être mise en relation avec les permis de séjour octroyés par certaines législations aux victimes de traite d'êtres humains lorsqu'elles portent plainte. La même compréhension doit s'exercer à l'égard des femmes qui ont rejoint un compagnon violent dans le cadre d'un regroupement familial, ou bien les victimes d'un mariage forcé. Si la disposition trouve sa source dans l'expérience des ONG d'aide aux victimes, elle procède aussi de l'obsession de déclenchement de la compétence pénale qui caractérise la convention. Car il existe des conditions d'octroi du permis au nombre desquelles l'obligation de la victime de coopérer avec les autorités dans le cas d'une enquête ou d'une procédure contre l'auteur. Toutefois, à l'heure où les politiques d'octroi de titre de séjour se tendent en Europe, l'Allemagne a déjà posé, avant même toute ratification, la seule réserve actuelle à ce texte en faisant savoir que, conformément aux possibilités ouvertes par l'article 78, elle n'appliquera pas l'article 59.

L'article 60 vise les demandes d'asile fondées sur le genre. Les Etats reconnaissent que les violences sexistes peuvent constituer une forme de « persécution » et donc motiver l'octroi de l'asile et même de la protection complémentaire ou subsidiaire : c'est-à-dire que la qualité de réfugiée ne serait pas reconnue aux femmes menacées de violences mais que leur renvoi serait impossible car il les exposerait à des violences fondées sur le genre « constituant un traitement inhumain ou dégradant ou menaçant la vie de l'individu ». Cette possibilité repose sur le fondement de la Convention européenne des droits de l'Homme ou de la directive " qualification " de l'Union européenne. Les femmes victimes bénéficient, en outre, du droit au non refoulement en vertu de l'article 33 de la convention de Genève de 1951, qui interdit de refouler les demandeurs d'asile dont le statut n'est pas encore établi, mais qui risquent la persécution en cas de renvoi.

Il convient de tempérer nettement cet optimisme. D'abord en rappelant que le Haut-Commissariat aux réfugiés note dans son rapport de 2011 une augmentation de $20 \%$ de demandes d'asiles vers les pays industrialisés, avec une hausse très nette dans le sud de l'Europe. Un tel contexte ne va guère dans le sens d'une compréhension plus développée. Ensuite parce que les Etats parties ne seront pas aiguillonnés par la jurisprudence de la Cour. Il est vrai qu'elle a déjà accepté de considérer que certains Etats mettent en danger des femmes, ce qui constitue une persécution fondée sur le genre. Ainsi une ressortissante afghane vivant en Suède a pu faire valoir que sa situation en instance de divorce et son mode de vie l'exposaient à des risques de traitement inhumains et dégradants en cas de renvoi (Cour EDH, 20 Juillet 2010, N. contre Suède).

Mais la jurisprudence est infiniment plus nuancée quand la Cour aborde les risques de mutilations génitales. S'il est établi à ses yeux que ces pratiques constituent des traitements inhumains et dégradants aux sens de l'article 3 de la Convention européenne des droits de l'Homme, elle se refuse néanmoins à suivre les requérantes dans leur raisonnement, dès qu'elle relève qu'elles disposent de moyens d'existence autonome et peuvent donc échapper aux pressions sociales et familiales (Cour EDH, Décision, 17 mai.2011, Izevbekhai c. Irlande). On peut en déduire que la convention ne sera probablement pas interprétée dans un sens très large quant aux demandes d'asile fondées sur le genre, qui sont souvent motivées par les risques d'excision.

La convention 210 a été ouverte à la ratification à Istanbul en 2011. Elle n'entrera en vigueur que si elle obtient dix ratifications dont au minimum huit de la part d'Etats membres du Conseil de l'Europe.

\section{BIBLIOGRAPHIE}

UN (data) ?? Assemblée générale des Nations unies, RES 65/208 2 août 2010, «Intensification de l'action menée pour éliminer toutes les formes de violence à l'égard des femmes. Rapport du secrétaire général ».

http://daccess-dds- ny.un.org/doc/UNDOC/GEN/N10/470/42/ PDF/N1047042.pdf?OpenElement (Récupéré le 6 avril 2012)

Assemblée parlementaire du Conseil de l'Europe, 2011, AVIS 280 (2011), Avis sur le projet de convention du Conseil de l'Europe sur la prévention et la lutte contre la violence à l'égard des femmes et la violence domestique.

http://assembly.coe.int/Documents/AdoptedText/ta11/ FOPI280.htm (récupéré le 23 mars 2012)

CAHVIO (data) / www.coe.int/t/dghl/standardsetting/ violence/meetings_fr.asp (récupéré le 23 mars 2012)

Conseil de l'Europe, 2000, Commission sur l'égalité des chances pour les femmes et les hommes Rapport «Violence à l'encontre des femmes en Europe » Doc. 866715 mars 2000, www. eurowrc.org > Contributions > Euro WRC Français (récupéré le 20 
mars 2012)

Conseil de l'Europe, 2002 , Rec. (2002) 5 du Conseil de l'Europe sur la Protection des femmes contre la violence (30 avril 2002) : https://wcd.coe.int/ViewDoc.jsp?id $=280925 \&$ Site $=$ CM (récupéré le 23 mars 2012)

Conseil de l'Europe, 2010, FLYER (mai 2010) :

www.coe.int/t/dghl/standardsetting/violence/Flyer_FR.pdf (récupéré le 20 mars 2 Conseil de l'Europe FLYER (mai 2010) 012)

Conseil de l'Europe Convention 210 du Conseil de l'Europe (2011) sur la prévention et la lutte contre la violence à l'égard des femmes et les violences domestiques conventions.coe.int/Treaty/ FR/Reports/Html/210.htm (récupéré le 23 mars 2012)

Cour EDH, 4 décembre 2003, M.C. contre Bulgarie (no 39272/98).

Cour EDH, 31 mai 2007, Kontrova c. Slovaquie (n 7510/04)

Cour EDH, 12 juin 2008, Bevacqua et S. c. Bulgarie (no $71127 / 01)$

Cour EDH, 9.juin.2009, Opuz c. Turquie (n 33401/02).

Cour EDH, 15.septembre.2009, E.S. et autres c. Slovaquie $\left(\mathrm{n}^{\circ}\right.$ 8227/04).

Cour EDH, 20 Juillet 2010, N contre Suède, (n $\left.{ }^{\circ} 23505 / 08\right)$.

Cour EDH, 14 octobre 2010, A. c. Croatie ( $\left.{ }^{\circ} 55164 / 08\right)$.

Cour EDH, Décision, 17 mai.2011, Izevbekhai c. Irlande $\left(\mathrm{n}^{\circ}\right.$ 43408/08)

Cour EDH, Décision, 20 septembre2011, Omeredo contre Autriche, (n 8969/10).

Haut-Commissariat aux réfugiés, 2012, Rapport 26 mars 2012, http://www.un.org/apps/newsFr/storyF. asp?NewsID $=27877 \& \mathrm{Cr}=$ asile $\& \mathrm{Cr} 1=$ (récupéré le 28 mars 2012)

PONCELLA P. et ROTH R, 2006 La fabrique du droit des sanctions pénales au Conseil de l'Europe, Perspectives de la justice, Documentation Française, Paris,

SUDRE F. (2008). Droit européen et international des droits de l'homme, Paris ? PUF.

SUDRE F., MARGUENAUD J.-P., ANDRIANTSIMBAZOVINA J., GOUTTENOIRE A., LEVINET M. (2007). Les grands arrêts de la Cour européenne des droits de l'Homme, Thémis, PUF, Paris.

W. H., 2012. "Convention sur la prévention et la lutte contre la violence à l'égard des femmes et la violence domestique ", Chronique des faits internationaux, Revue Générale de Droit international Public

Recebido: 28/06/2013

Última revisão: 30/05/2014

Aceite final: 07/06/2014 